\title{
Tracheal resection with end-to-end anastomosis for post-intubation cervical tracheal stenosis: study of 14 cases
}

\author{
R NANDAKUMAR, C JAGDISH, C B PRATHIBHA, C SHILPA, V SREENIVAS, \\ A M BALASUBRAMANYA, R C NAYAR
}

Department of Otorhinolaryngology and Head and Neck Surgery, St John's Medical College and Hospital, Bangalore, India

\begin{abstract}
Background and objectives: The incidence of acquired laryngeal stenosis is increasing. This retrospective study aimed to assess the long term results of circumferential resection with end-to-end tracheal anastomosis for isolated post-intubation stenosis of the cervical trachea, and to review the relevant literature.

Methods: Twelve male and two female patients (aged 16-30 years, mean age 24 years) treated between February 2003 and December 2008 were included. Hospital and office records were reviewed and relevant surgical details recorded.

Results: Indications for tracheal resection anastomosis were post-intubation stenosis ( 78.57 per cent) and trauma (21.42 per cent). One to five tracheal rings were resected (i.e. $1-2.5 \mathrm{~cm}$ of cervical trachea). Tracheal anastomosis was considered successful if the patient remained asymptomatic for 24 months of close follow up (involving regular flexible bronchoscopy and neck X-ray). The anastomotic success rate was 92.85 per cent.

Conclusion: Tracheal resection and end-to-end anastomosis is relatively safe and reliable for definitive treatment of benign tracheal stenosis in appropriate patients. Local application of mitomycin C prevents granulation and aids long term airway patency.
\end{abstract}

Key words: Tracheal Stenosis; Intubation, Endotracheal; Anastomosis, Surgical

\section{Introduction}

Laryngotracheal stenosis is becoming a common clinical challenge for otolaryngologists and head and neck surgeons, due to the increased incidence of acquired laryngeal stenosis. Acquired laryngeal stenosis in adults and children is usually a complication of prolonged intubation, or due to external trauma. ${ }^{1}$ Prolonged ventilation, together with advances in critical care medicine, cardiopulmonary surgery and neurosurgery, have resulted in an increased number of intubation-related laryngotracheal injuries. ${ }^{2}$ Within the trachea, trauma at the tracheostomy site, or around the cuff or tip of the ventilation tube, may result in granulation tissue formation. Following the onset of granuloma at the injury site, the reparative process may lead to the formation of fibrous tissue, with a compromised airway. $^{3}$

Treatment options for tracheal stenosis include laser endoscopy, endoscopic dilatation, interposition grafting, tracheal stenting and tracheal resection. Each has its particular advantages and disadvantages. Endoscopic dilatation is usually performed as a temporary measure prior to definitive therapy. Restenosis may develop within days to weeks. Laser vaporisation and radial division of tracheal strictures have also been reported. Laser treatment is most successful for thin, web-like stenotic lesions (which are rare); in thicker lesions, laser treatment may worsen the severity of the lesion. Endoscopic dilatation and laser vaporisation are less successful in cases of stenosis that is circumferential, without cartilaginous support or longer than $1 \mathrm{~cm} .{ }^{4}$ Tracheal stents have been used as an alternative or complement to surgery. Tracheotomy is another acceptable, life-long management option for tracheal stenosis patients who are poor surgical risks. Tracheal resection is well established as the definitive treatment for benign tracheal stenosis. Several series attest to its success in carefully selected patients. ${ }^{5-7}$

The current study aimed to assess the long term results of circumferential resection with end-to-end tracheal anastomosis, performed to treat isolated postintubation stenosis of the cervical trachea. We focussed on the anastomotic success rate, morbidity and postoperative course. The study reviewed 14 cases of 
tracheal resection with end-to-end anastomosis performed between February 2003 and December 2008.

\section{Materials and methods}

A retrospective review was conducted of 14 patients who had undergone circumferential tracheal resection with end-to-end anastomosis between February 2003 and December 2008. These patients comprised 12 males and two females aged between 16 and 30 years (mean age, 24 years).

We excluded from the study any patients who had undergone tracheal resection for tracheal or thyroid neoplasm, or who had true subglottic (cricoid) stenosis.

Hospital and clinic records were reviewed for each patient. We recorded details of each patient's clinical presentation, intubation and/or tracheostomy history, grade and length of stenosis, and surgical procedure (Table I). Follow-up information was obtained by direct patient contact and from clinic records. Followup data were obtained for all patients from 24 months to five years post-treatment. Treatment was considered successful if patients were asymptomatic for a minimum follow-up period of 24 months.

\begin{tabular}{|c|c|c|}
\hline \multicolumn{2}{|c|}{ PATIENT DETAILS } & \\
\hline Parameter & Value & $\%$ \\
\hline \multicolumn{3}{|l|}{$\operatorname{Sex}(n)$} \\
\hline - Male & 12 & 85.71 \\
\hline - Female & 2 & 14.28 \\
\hline \multicolumn{3}{|l|}{ Age (years) } \\
\hline - Range & $16-30$ & \\
\hline - Mean & 24 & \\
\hline \multicolumn{3}{|l|}{ Cause of intubation $(n)$} \\
\hline - Organophosphate poisoning & 11 & 78.57 \\
\hline - Road traffic accident & 1 & 7.14 \\
\hline - Trauma & 2 & 14.28 \\
\hline \multicolumn{3}{|l|}{ Intubation duration } \\
\hline - Endotracheal intubation range (days) & $1-21$ & \\
\hline - Endotracheal intubation mean (days) & 10 & \\
\hline - Tracheostomy dependence $(n)$ & 14 & 100 \\
\hline \multicolumn{3}{|l|}{ Prior treatment $(n)$} \\
\hline - Oral steroids & 14 & 100 \\
\hline - Montgomery $\mathrm{T}$ tube insertion & 3 & 21.42 \\
\hline \multicolumn{3}{|l|}{ Stenosis location $(n)$} \\
\hline - Cervical trachea & 14 & 100 \\
\hline \multicolumn{3}{|l|}{ Stenosis grade* $(n)$} \\
\hline$-1(<70 \%)$ & 0 & 0 \\
\hline$-2(70-90 \%)$ & 6 & 42.81 \\
\hline$-3(>90 \%)$ & 6 & 42.81 \\
\hline-4 (No lumen seen) & 2 & 14.28 \\
\hline \multicolumn{3}{|l|}{ Stenosis length $(\mathrm{cm})$} \\
\hline - Range & $1-5$ & \\
\hline - Mean & 3.21 & \\
\hline \multicolumn{3}{|l|}{ Stenosis type $(n)$} \\
\hline - Circumferential & 13 & 92.85 \\
\hline - Anterior & 1 & 7.14 \\
\hline \multicolumn{3}{|l|}{ Anastomosis details $(n)$} \\
\hline - Cricoid to 3rd tracheal ring & 1 & 7.14 \\
\hline - Cricoid to 4 th tracheal ring & 1 & 7.14 \\
\hline - Cricoid to 5th tracheal ring & 3 & 21.42 \\
\hline - Cricoid to 6 th tracheal ring & 2 & 14.28 \\
\hline-1 st to 5 th tracheal ring & 5 & 35.71 \\
\hline-2 nd to 4 th tracheal ring & 1 & 7.14 \\
\hline-2 nd to 6 th tracheal ring & 1 & 7.14 \\
\hline
\end{tabular}


posterior midline and moving laterally at $5 \mathrm{~mm}$ intervals on both sides up to the anterior trachea. Once the posterior sutures were placed, they were tied simultaneously from both sides with knots lying within the tracheal lumen. The neck was flexed, and the distal endotracheal tube was removed. Following this, the oral endotracheal tube was passed beyond the anastomotic site for ventilation. The lateral and the anterior sutures, which had already been placed through the cartilage, were then tied with knots lying outside the tracheal lumen. The suture line was tested for any air leak by submerging it in saline.

The strap muscles were then re-approximated in the midline. Platysma and skin were closed in layers, after placement of a closed suction drain. A Grillo stitch using Prolene 1-0 was placed between the chin and the manubrium to prevent the patient from extending the neck in the post-operative period.

All patients were extubated immediately after the procedure, while still in the operating theatre. The Grillo stitch was maintained for 21 days post-operatively.

A soft tissue lateral neck X-ray was taken at one, three, six, 12, 18 and 24 months post-operatively. Bronchoscopy was performed at three, six, 12, 18 and 24 months post-operatively. Morbidity and tracheal anastomosis success rates were assessed clinically. The tracheal anastomosis was considered successful if the patient had remained asymptomatic for 24 months of close follow up (involving regular flexible bronchoscopy in the clinic and lateral neck X-ray demonstrating a patent airway).

\section{Results}

At the termination of the surgical procedure, all 14 patients could ventilate freely after extubation.

One patient required re-intubation on the sixth day after extubation, followed by a tracheostomy on the eight day, due to acute ascending viral transverse myelitis.

In another patient, we encountered right carotid artery bleeding, which was controlled successfully. This patient also developed an iatrogenic right vocal fold paralysis.

Nasogastric feeding was commenced in 14 patients within 24 hours post-operatively. Oral intake was tolerated well by the 10th post-operative day. The length of hospitalisation ranged from 22 to 30 days, with a mean stay of 24 days. No post-operative dysphagia or respiratory difficulty was encountered over two years' follow up. The follow-up period ranged from two to four years.

The anastomosis success rate was seen in $13(92.85$ per cent) out of 14 patients.

\section{Discussion}

Treatment options for tracheal stenosis include laser endoscopy, endoscopic dilatation, interposition grafting, tracheal stenting and tracheal resection. Each has its particular advantages and disadvantages. ${ }^{4}$ Therefore, careful pre-operative evaluation of tracheal resection candidates is essential. Factors such as a previous failed resection, higher level of stenosis, and stenosis involving more than 50 per cent of the tracheal length have been observed to negatively influence surgical outcomes. ${ }^{6}$ Tracheal stenting is usually considered to be a better option for patients who are poor candidates for tracheal resection, due to previous conditions or comorbidity which increases their surgical risk.

Tracheal resection is well established as the definitive treatment for benign tracheal stenosis. Several series attest to its success in carefully selected patients. ${ }^{5-7}$ In our series of 14 patients who underwent resection and anastomosis, we achieved a success rate of 92.85 per cent.

Experimental data have emphasised the fact that the primary requirement for successful end-to-end tracheal anastomosis is the creation of a tension-free anastomosis line. ${ }^{5}$ According to Montgomery, tension should not exceed $1000 \mathrm{~g}$ at the suture line. ${ }^{10}$ Numerous mobilisation techniques have been used to gain tracheal length, including pretracheal and peritracheal dissection, suprahyoid release, and anterior mediastinal dissection. Extreme neck flexion, aided by a chin-tochest suture, also contributes to a tension-free anastomosis. ${ }^{11}$ Peskind et al. recently advocated the routine use of a supralaryngeal release procedure (combining infrahyoid and suprahyoid elements) to decrease tension at the level of the anastomotic suture line. ${ }^{7}$ However, in their series they noted that this procedure fixed the larynx in an abnormally low position, preventing elevation of the larynx during swallowing and thus producing post-operative dysphagia in all patients.

On the other hand, various reports have proposed that a tracheal defect of $2 \mathrm{~cm}$ or less can be successfully managed by primary anastomosis alone, without release techniques. ${ }^{10,12,13}$

In our series, resection varied from one to five tracheal rings, resulting in resection of 1 to $2.5 \mathrm{~cm}$ of the cervical trachea (there are approximately two tracheal rings per centimetre of trachea). ${ }^{14} \mathrm{~A}$ laryngeal release procedure was performed in five cases at our centre. Revision end-to-end tracheal resection was not required in our series, which achieved an overall success rate of 92.85 per cent.

Our results suggest that a tracheal defect of $2 \mathrm{~cm}$ or less can be successfully managed by primary anastomosis alone, without release techniques. This conclusion is in agreement with the cadaver study of Laccourreye and Bessede, who noted that after resection of three to six tracheal rings, the intertracheal ring ligament elasticity allowed for tracheal end-to-end anastomosis without any adjunctive measures other than mediastinal tracheal dissection and wide, blunt cervical dissection between the sternohyoid and thyrohyoid-sternothyroid muscles. ${ }^{15}$

Granulation tissue at the anastomotic site is the most common complication, and is more common with the use of non-absorbable sutures. ${ }^{11}$ Grillo et al. noted that significantly fewer patients developed granulation tissue at the site of tracheal anastomosis after the suture material was changed from Tevdek to Vicryl. ${ }^{6}$ Placing the anastomotic sutures through healthy 
tissue extramucosally, along with application of mitomycin $\mathrm{C}$ at the anastomotic site, also decreases the likelihood of granulation tissue development. Mitomycin C is an antineoplastic antibiotic with antiproliferative effects on fibroblasts, via inhibition of DNA synthesis (it leads to cross-linkage of strands of the double helix, inhibiting DNA replication). It can cause fibroblast arrest without sacrificing re-epithelialisation. ${ }^{16,17}$ Local application of mitomycin $\mathrm{C}$ to treat tracheal granulations has been documented. ${ }^{18}$ Our results suggest that local application of mitomycin $\mathrm{C}$ can be used to prevent tracheal granulations.

All our patients were extubated immediately after the surgical procedure. The timing of extubation has been a subject of debate. Several authors support leaving the patient intubated for 24 to 48 hours, to allow air-tight closure prior to the introduction of positive intratracheal pressure. ${ }^{7}$ In our opinion, maintaining an endotracheal tube presents a risk of injury to the anastomosis or a different segment of the trachea. Therefore, in our department extubation was always performed in the operating theatre. In agreement with Grillo et al., we successfully performed immediate extubation in all our patients. ${ }^{6}$

- In this study, 14 patients with cervical tracheal stenosis underwent successful circumferential resection and end-to-end tracheal anastomosis

- Five patients underwent suprahyoid release due to resection of more than four tracheal rings; this gained tracheal length and thus reduced anastomosis tension

- Mitomycin $\mathbf{C}$ was applied at the anastomosis site to prevent granulation tissue formation

- Tracheal resection and anastomosis is a relatively safe, reliable procedure for benign cervical tracheal stenosis in appropriate patients

Potential post-operative complications following tracheal end-to-end anastomosis include airway obstruction, infection of the operated region, innominate artery rupture and dysphagia. ${ }^{7}$ None of these complications were noted in our series, except transient dysphagia lasting for up to 10 days. Iatrogenic complications included unilateral vocal fold paralysis in one patient.

\section{Conclusion}

Tracheal resection with end-to-end anastomosis is a relatively safe and reliable procedure for the definitive treatment of benign tracheal stenosis in appropriately selected patients. In our series, we had a 92.85 per cent anastomotic success rate, and no deaths occurred as a result of the procedure.

We conclude that tracheal resection with end-to-end anastomosis achieves wound healing by primary intention, and has a low complication rate and high success rate. In patients in whom one to four tracheal rings are resected, our findings suggest that end-toend tracheal anastomosis does not require the systematic use of adjunctive measures to release tension at the level of the suture line. In our series, local application of mitomycin $\mathrm{C}$ prevented the development of granulations and resulted in long term airway patency.

\section{References}

1 Cotton RT. Management of subglottic stenosis. Otolaryngol Clin North Am 2000;33:111-30

2 Montgomery WW. Tracheal stenosis. In: Montgomery WW, ed. Surgery of the Upper Respiratory System, 2nd edn. Philadelphia: Lea \& Febiger, 1989;2:455-520

3 Grillo HC, Mathisen DJ. Surgical management of tracheal strictures. Surg Clin North Am 1988;68:511-24

4 Ossoff RH, Tucker GF Jr, Duncavage JA, Toohill RJ. Efficacy of bronchoscopic carbon dioxide laser surgery for benign strictures of trachea. Laryngoscope 1985;95:1220-3

5 Anand VK, Alemar G, Warren ET. Surgical considerations in tracheal stenosis. Laryngoscope 1992;102:237-43

6 Grillo HC, Donahhue DM, Mathisen DJ, Wain JC, Wright CD. Post intubation tracheal stenosis. Treatment and results. J Thorac Cardiovasc Surg 1995;109:486-93

7 Peskind SP, Stanley RB Jr, Thangathurai D. Treatment of the compromised trachea with sleeve resection and primary repair. Laryngoscope 1993;103:203-11

8 Pearson FG, Cooper JD, Nelems JM, Van Nostrand AW. Primary tracheal anastomosis after resection of the cricoid cartilage with preservation of the recurrent laryngeal nerves. J Thorac Cardiovasc Surg 1975;70:806-16

9 Grillo HC, Mathisen DJ, Ashiku SK, Wright CD, Wain JC. Successful treatment of idopathic laryngotracheal stenosis by resection and primary anastomosis. Ann Otol Rhinol Laryngol 2003;112:798-800

10 Montgomery WW. Suprahyoid release for tracheal anastomosis. Arch Otolaryngol 1974;99:255-60

11 Wynn R, Harel G, Lim JW. Tracheal resection with end to end anastomosis for benign tracheal stenosis. Ann Otol Rhinol Laryngol 2004;113:613-17

12 Greenberg SD. Tracheal reconstruction: an experimental study. Arch Otolaryngol 1960;72:565-74

13 Montgomery WW. Reconstruction of the cervical trachea. Ann Otol Rhinol Laryngol 1964;73:5-15

14 Weber AL, Grillo HC. Tracheal stenosis. An analysis of 151 cases. Radiol Clin North Am 1978;16:291-308

15 Laccourreye H, Bessede JP. An anatomical study of tracheal resections including pulmonary sequelae [in French]. Ann Otolaryngol Chir Cervicofac 1981;98:425-30

16 Stewart CE 4th, Kim JY. Application of mitomycin-C for head and neck keloids. Otolaryngol Head Neck Surg 2006;135: 946-50

17 Viera MH, Amini S, Valins W, Berman B. Innovative therapies in the treatment of keloids and hypertrophic scars. Clin Aesthet Dermatol 2010;3:20-6

18 Mizuno Y, Sasazuki M, Aibe M, Honjo S. Study on the etiology and treatment of tracheal granulation tissue in patients with severe motor and intellectual disabilities [in Japanese]. No Tu Hattatsu 2009; 41:275-8

Address for correspondence:

Dr R Nandakumar,

Assistant Professor,

Department of Otolaryngology Head and Neck Surgery,

St John's Medical College Hospital,

Koramangala,

Bangalore 560 034, India

E-mail: nandakumarrajan@yahoo.com

Dr R Nandakumar takes responsibility for the integrity of the content of the paper

Competing interests: None declared 